\title{
Synthesis of a sucrose dimer with enone tether; a study on its functionalization
}

\author{
Zbigniew Pakulski, Norbert Gajda, Magdalena Jawiczuk, Jadwiga Frelek, \\ Piotr Cmoch and Sławomir Jarosz ${ }^{*}$
}

\author{
Full Research Paper \\ Address: \\ Institute of Organic Chemistry, Polish Academy of Sciences, ul. \\ Kasprzaka 44/52, 01-224 Warsaw, Poland \\ Email: \\ Sławomir Jarosz* - slawomir.jarosz@icho.edu.pl \\ * Corresponding author \\ Keywords: \\ CD-spectroscopy; Cotton effect; multivalent glycosystems; \\ osmylation; stereoselective synthesis; sucrose
}

Open Access

\author{
Beilstein J. Org. Chem. 2014, 10, 1246-1254. \\ doi:10.3762/bjoc. 10.124 \\ Received: 05 March 2014 \\ Accepted: 30 April 2014 \\ Published: 28 May 2014 \\ This article is part of the Thematic Series "Multivalent glycosystems for \\ nanoscience". \\ Guest Editor: A. Casnati \\ (C) 2014 Pakulski et al; licensee Beilstein-Institut.
}

License and terms: see end of document.

\begin{abstract}
The reaction of appropriately functionalized sucrose phosphonate with sucrose aldehyde afforded a dimer composed of two sucrose units connected via their C6-positions ('the glucose ends'). The carbonyl group in this product (enone) was stereoselectively reduced with zinc borohydride and the double bond (after protection of the allylic alcohol formed after reduction) was oxidized with osmium tetroxide to a diol. Absolute configurations of the allylic alcohol as well as the diol were determined by circular dichroism (CD) spectroscopy using the in situ dimolybdenum methodology.
\end{abstract}

\section{Introduction}

Molecular recognition is one of the most important phenomena in stereoselective processes. Chiral crown ethers (or analogs) are particularly useful in enantioselective reactions [1,2] as well as differentiation of chiral guests [3,4]. From all of the chiral platforms designed for such receptors, sugars are the most promising due to their availability and biocompatibility. Up to date only monosaccharides have found a wide application in the synthesis of crown ether analogs $[5,6]$. The disaccharide scaffold is much less pronounced [7].
During the past decade we have become engaged in the preparation of the analogs of crown and aza-crown ethers with sucrose scaffold. It is based on a selective protection of 1',2,3,3',4,4'hexa- $O$-benzylsucrose (1) either at the glucose (C-6) [8] or fructose (C-6') [9] end and further transformations to a variety of macrocycles (2-4; Figure 1). Such receptors exhibit interesting complexing properties towards chiral ammonium salts including amino acids [10-14]. More complex sucrose macrocycles, such as $\mathbf{5}$, are available, although in rather low yield [15]. 


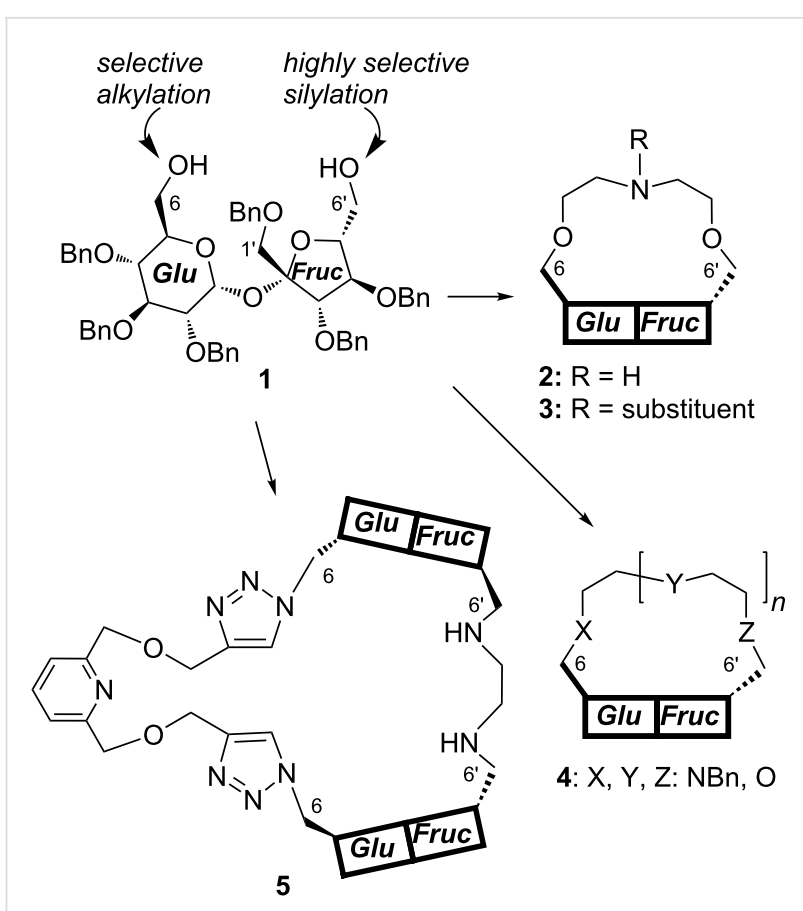

Figure 1: Examples of sucrose-based macrocycles.

In this paper we present an approach to other derivatives containing two sucrose units. This type of dimers may be eventually used for the construction of macrocycles by (simple) connecting their C-6' (fructose) ends.

\section{Results and Discussion}

Coupling of two sugar units can be performed by a number of methods. The best one in our hands was the Wittig-type methodology shown in Figure 2. The properly activated sugar is converted into phosphorane or phosphonate which - upon reaction with an aldehyde derived from another monosaccharide provides higher carbon sugar (HCS) enone [16-18].
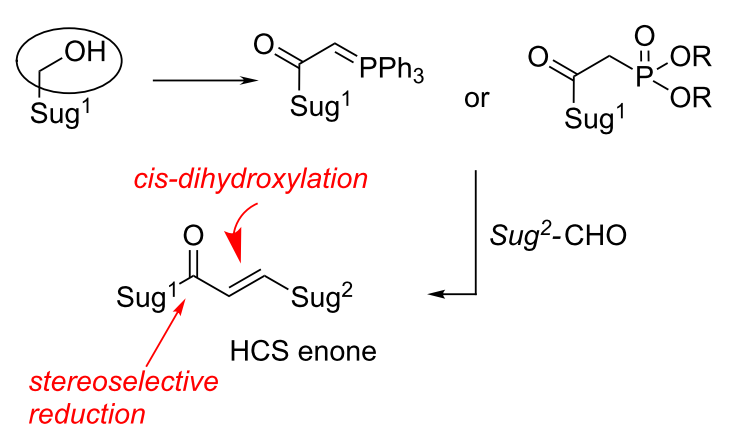

Figure 2: Synthesis of higher sugar precursors by a Wittig-type methodology.

Application of this methodology to selectively protected $2,3,3^{\prime}, 4,4^{\prime}$-penta- $O$-benzylsucrose allowed us to elongate the parent disaccharide at either terminal position ( $\left(1^{\prime}, 6^{\prime}\right.$, and $\left.6^{\prime}\right)$ by relatively small (C2 or $\mathrm{C} 7)$ unit and prepare so-called higher sucroses in good yields $[19,20]$. A similar approach is used now for a more convenient hexa- $O$-benzyl derivative which is easily silylated at the 'fructose end' providing alcohol 6 [8]. This alcohol was converted into aldehyde 7 [21] (route a in Scheme 1) and separately into phosphonate 9 (route b). Reaction of both synthons under the mild PTC conditions [22-24] afforded the respective enone $\mathbf{1 0}$ in good yield (Scheme 1).

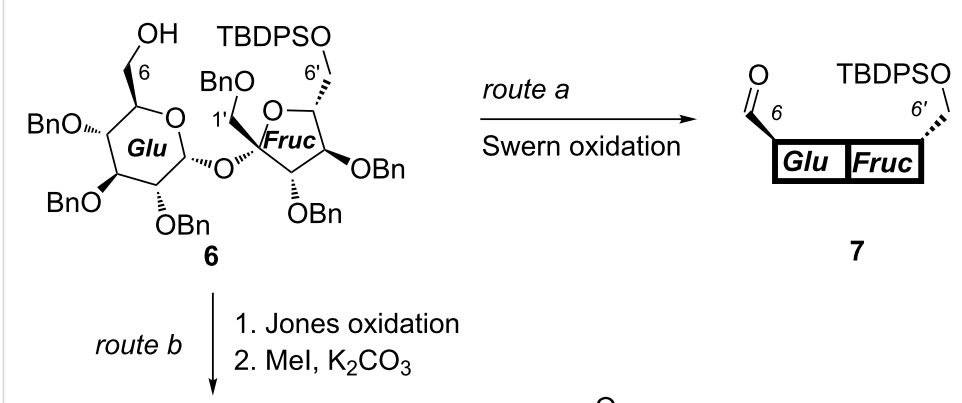

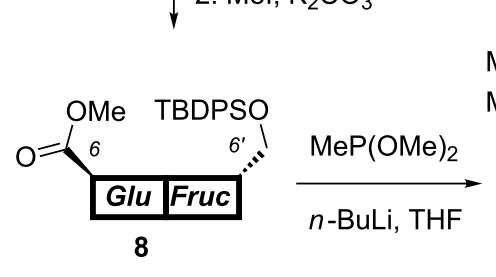

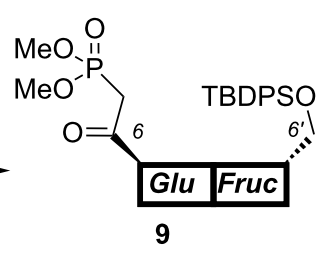

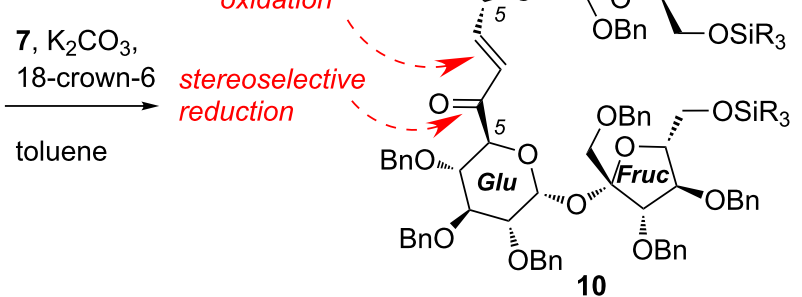


Functionalization of the three-carbon atom unit connecting the C5-positions of both sucrose units required reduction of the carbonyl group of the enone system and oxidation of the double bond.

We have already reported that reduction of higher carbon sugar enones of the D series with zinc borohydride is highly selective and provides the corresponding allylic alcohols with the $R$ configuration at the newly created stereogenic center, as the only products. This can be rationalized assuming the cyclic model of such reduction [25] shown in Scheme 2. We expected, therefore, also very high selectivity in the reduction of $\mathbf{1 0}$.

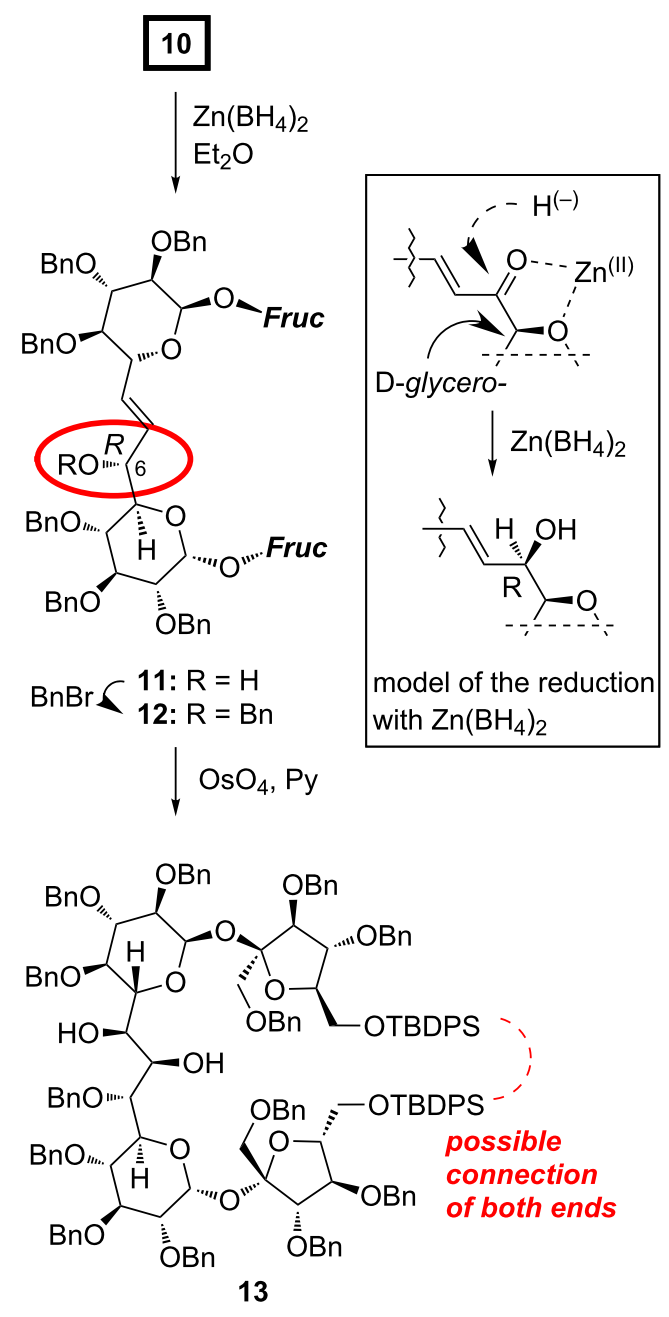

Scheme 2: Synthesis of the diol 13 containing two sucrose units

Indeed, treatment of enone $\mathbf{1 0}$ with $\mathrm{Zn}\left(\mathrm{BH}_{4}\right)_{2}$ under the standard conditions afforded allylic alcohol $\mathbf{1 1}$ as single stereoisomer in $65 \%$ yield. Based on our model, the $R$-configuration might be safely assigned to the new stereogenic center. This assignment was further verified independently by circular dichroism spectroscopy (CD) using the in situ dimolybdenum methodology (see next chapter).

Next steps of the synthesis consisted of the protection of the $\mathrm{C} 6-\mathrm{OH}$ as benzyl ether (to 12) and osmylation of the double bond. The cis-dihydroxylation provided, as single stereoisomer, a diol to which structure $\mathbf{1 3}$ could be assigned on the basis of the Kishi rule [26] (Scheme 2). It postulates that the attack of $\mathrm{OsO}_{4}$ occurs from the side opposite to hydroxy (alkoxy) substituent(s) flanking the double bond. Since in 12, both alkoxy units act in the same direction, very high diastereoselectivity is not surprising. The assignment of the configuration of this diol was further confirmed also by the CD methodology; this is discussed in the next chapter.

\section{Determination of the absolute configuration of 11 and 13}

It is widely acceptable that the circular dichroism (CD) spectroscopy utilizing the in situ dimolybdenum methodology offers the hard proof of the absolute configuration of the vic-diols [2729]. In this methodology, dimolybdenum tetraacetate acts as auxiliary chromophore allowing the application of electronic circular dichroism (ECD) to (otherwise in ECD non-observable) vic-diols. $\mathrm{Mo}_{2}(\mathrm{OAc})_{4}$ when mixed with a chiral diol ligand forms complexes active in ECD in which a transfer of ligand chirality to the in situ-formed complex occurs in solution. Thus, stereochemistry of vic-diols can be easily assigned based on the helicity rule developed for this class of compounds. This rule correlates the positive/negative signs of Cotton effects (CE) occurring in the 300-400 $\mathrm{nm}$ spectral range in the ECD spectra with the positive/negative sign of the $\mathrm{O}-\mathrm{C}-\mathrm{C}-\mathrm{O}$ torsion angle of the diol unit of resultant complexes with the $\mathrm{Mo}_{2}$-core. The basic assumption leading to the assignment of the absolute configuration (AC) based only on the ECD spectra with the $\mathrm{Mo}_{2}-$ core preferring the gauche conformation of the diol units with both $\mathrm{O}-\mathrm{C}-\mathrm{C}-\mathrm{C}$ fragments in an antiperiplanar arrangement (Figure 3). This arrangement is favored, for steric reasons, i.e., to avoid any interaction with the carboxylate ligands remaining in the stock complex. As a result of the structure-ECD spectra relationship, it is possible to assign the $\mathrm{AC}$ of the diol moiety unambiguously on the basis of the ECD spectra alone.

In the past few years, this simple but, above all, efficient and effective method is becoming more and more recognized as evidenced by the steadily increasing number of reports in the literature about its successful application in the determination of the AC of 1,2-diols [30-32].

Therefore, in assignment of the AC of compounds under the present study (13, 14 and 16), we decided just to take advantage of the in situ methodology. 


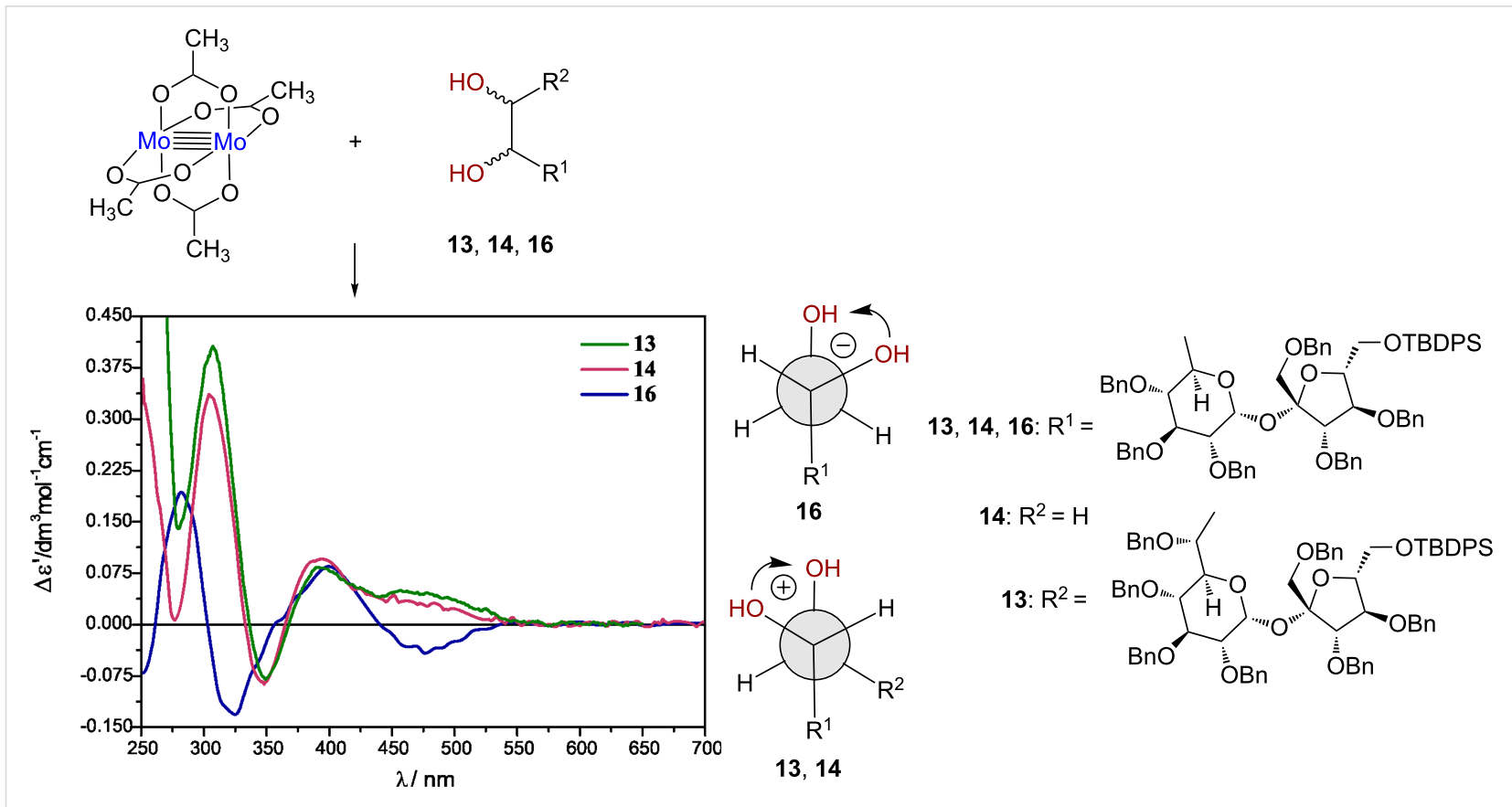

Figure 3: CD spectra of in situ formed chiral complexes of 13 (green line), 14 (purple line) and 16 (blue line) with dimolybdenum tetraacetate recorded in DMSO; right: preferred gauche conformation of the diols 13,14 and 16 in the complex with $\mathrm{Mo}_{2}$-core.

This method was used to prove indirectly the $6 R$ configuration at the newly created stereogenic center in allylic alcohol $\mathbf{1 1}$. The double bond in $\mathbf{1 1}$ was cleaved with ozone and the resulting ozonide was reduced with $\mathrm{NaBH}_{4}$; this sequence afforded sucrose vic-diol 14 and (as a byproduct) sucrose alcohol 6 (Scheme 3).

The positive sign of the Cotton effect at around $307.0 \mathrm{~nm}$ recorded for the complex of the diol 14 with $\mathrm{Mo}_{2}(\mathrm{OAc})_{4}$ unambiguously pointed at the $6 R$ configuration (Figure 3 ). However, to exclude any errors we have also prepared 14 and epimeric alcohol 16; its synthesis is shown in Scheme 3.

First, aldehyde 7 was converted into olefin 15 by treatment with the simplest Wittig reagent: $\mathrm{Ph}_{3} \mathrm{P}=\mathrm{CH}_{2}$. Subsequent osmylation of the double bond in $\mathbf{1 5}$ provided two stereoisomeric diols in a 1:1 ratio; the first one was identical in all respects with the diol obtained from degradation of $\mathbf{1 1}$.

The resultant ECD spectra of the $\mathrm{Mo}_{2}$-core with compounds 13 and $\mathbf{1 6}$ are shown in Figure 3. Based on the positive CE's at $308.5 \mathrm{~nm}$ for $\mathbf{1 3}$ and negative at $310 \mathrm{~nm}$ for $\mathbf{1 6}$, respectively, the positive (negative) sign of the $\mathrm{O}-\mathrm{C}-\mathrm{C}-\mathrm{O}$ torsion angle has been attributed to these diols. In the next step, based on the preferred gauche conformation of the diol unit with both $\mathrm{O}-\mathrm{C}-\mathrm{C}-\mathrm{C}$ fragments in an antiperiplanar arrangement as shown in Figure 3, we were able to assign unambiguously the $(7 R, 8 R) \mathrm{AC}$ to diol $\mathbf{1 3}$ and $(6 S)$ to $\mathbf{1 6 .}$

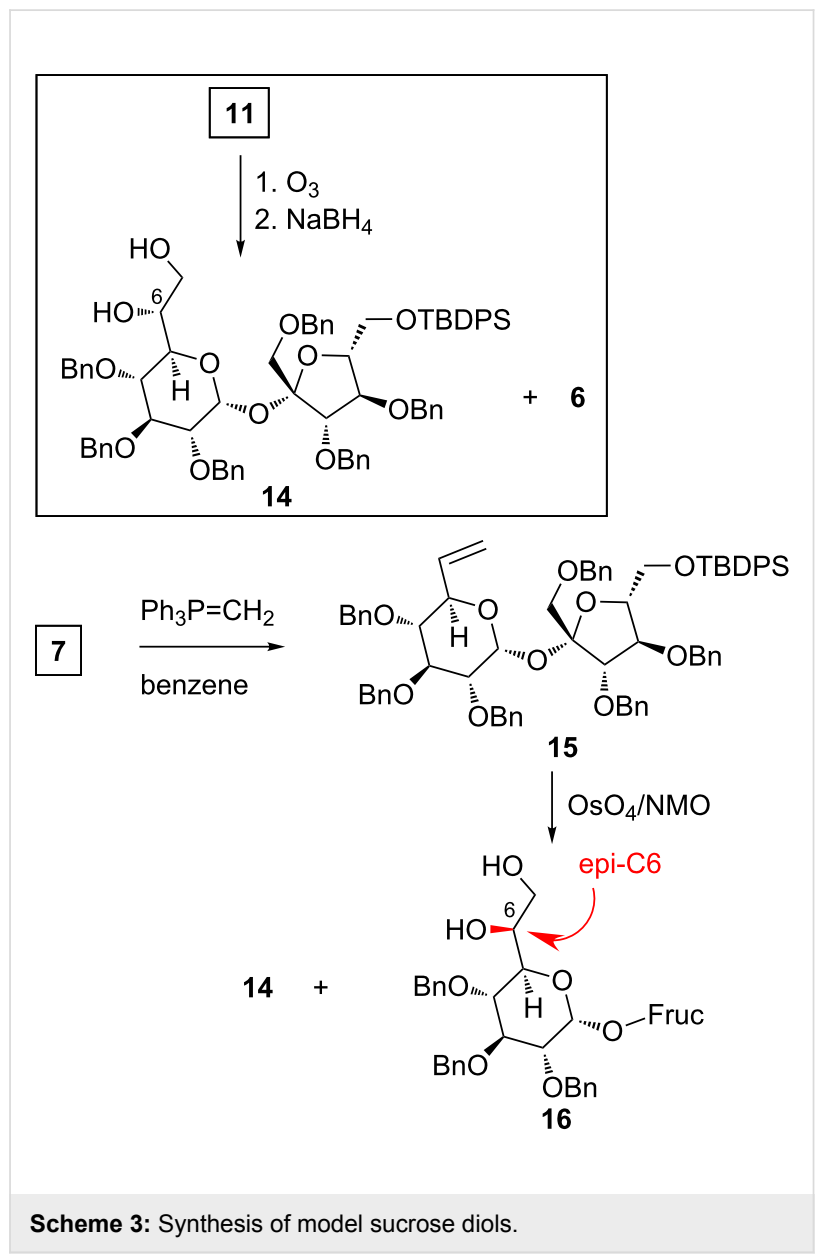




\section{Conclusion}

Coupling of two properly activated sucrose sub-units afforded the dimer in which both glucose-rings were connected via an enone linker. The dimer was then converted into a (partially protected) triol via a stereoselective reduction of the carbonyl group and highly selective cis-dihydroxylation of the double bond. The configuration at each new stereogenic center was determined by $\mathrm{CD}$ spectroscopy using the so-called dimolybdenum methodology which allows for fast, easy, and effective assignment of the absolute configuration of vic-diols. We have confirmed the usefulness of this simple methodology which can be applied even in cases when other spectroscopic methods fail.

It is worthy to point out that this methodology, which is used in the synthesis of more simple derivatives such as higher carbon sugars, was also applicable for the preparation of the sucrose dimer.

\section{Experimental}

\section{General methods}

All reported NMR spectra were recorded with a Varian-Vnmrs$600 \mathrm{MHz}$ spectrometer (at 600 and $150 \mathrm{MHz}$ for ${ }^{1} \mathrm{H}$ and ${ }^{13} \mathrm{C}$ NMR spectra, respectively) for solutions in $\mathrm{CDCl}_{3}$ at room temperature. Chemical shifts $(\delta, \mathrm{ppm})$ were determined relative to TMS as the internal standard. Most of the resonances were assigned by COSY $\left({ }^{1} \mathrm{H}-{ }^{1} \mathrm{H}\right)$ and gradient selected HSQC and HMBC correlations. Mass spectra were recorded with an ESI/ MS Mariner (PerSeptive Biosystem) mass spectrometer. Elemental analyses were obtained using a Perkin-Elmer 2400 $\mathrm{CHN}$ analyzer. Optical rotations were measured with a Jasco $\mathrm{P}-2000$ digital polarimeter for solutions in $\mathrm{CHCl}_{3}(c=0.3)$ at room temperature. Flash and column chromatographic separations were performed on silica gel (Merck, 230-400 mesh). Progress of the reactions was monitored by thin-layer chromatography (TLC) performed on aluminum plates covered with silica gel (60 F254, Merck).

The ECD spectra were acquired at room temperature in DMSO (for UV-spectroscopy, Fluka) on a Jasco J-715 spectropolarimeter and were collected at $0.5 \mathrm{~nm} / \mathrm{step}$ with an integration time of $0.25 \mathrm{~s}$ over the range $235-800 \mathrm{~nm}$ with $200 \mathrm{~nm} / \mathrm{min}$ scan speed, 5 scans. For the ECD standard measurements the chiral diols ( $\sim 3.6 \mathrm{mg}$, ca. $0.003 \mathrm{M})$ was mixed with stock complex $\left[\mathrm{Mo}_{2}\left(\mathrm{O}_{2} \mathrm{CCH}_{3}\right)_{4}\right](\mathrm{Mo} 1)(\sim 0.9 \mathrm{mg}$, ca. $0.002 \mathrm{M})$ and dissolved in DMSO $(1 \mathrm{~mL})$ so that the molar ratio of the stock complex to ligand was about 1:1.5 in general. Quantitative values could not be obtained with the in situ dimolybdenum method since the concentration of the chiral complex formed in solution and its actual structure were unknown. So the ECD data are given as the $\Delta \varepsilon^{\prime}$ values, which are calculated in the usual manner by means of the equation $\Delta \varepsilon^{\prime}=\Delta A / c \times d, A$ being the absorption, $c$ the molar concentration of the chiral ligand, assuming $100 \%$ complexation, and $d$ the path length of the cell.

The numbering of the atoms in sucrose dimers is as followed. The bottom part (green) is marked A and the top marked as B. The original numbering of the sucrose skeleton in both parts is retained (i.e., the glucose part is numbered $\mathrm{C} 1-\mathrm{C} 6$ and fructose C1'-C6')

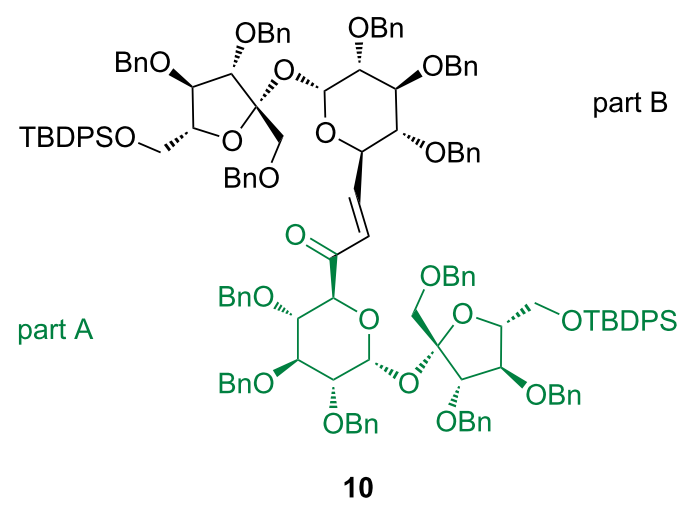

Synthesis of phosphonate 9. To a cooled $-78{ }^{\circ} \mathrm{C}$ solution of dimethyl methylphosphonate $(110 \mu \mathrm{L}, 1.0 \mathrm{mmol})$ in THF $(7 \mathrm{~mL})$ a $2.5 \mathrm{M}$ solution of butyllithium in hexane $(0.4 \mathrm{~mL}$, $1.0 \mathrm{mmol}$ ) was added and the mixture was stirred for $15 \mathrm{~min}$. Then, a solution of 8 (325 mg, $0.28 \mathrm{mmol})$ in THF ( $5 \mathrm{~mL})$ was slowly added and the mixture was stirred for additional $30 \mathrm{~min}$. Reaction was quenched by addition of a saturated solution of $\mathrm{NaCl}$ (5 drops). The mixture was concentrated, and the residue was purified by column chromatography (hexane-ethyl acetate, $10: 1 \rightarrow 1: 1)$ to afford title compound $9(227 \mathrm{mg}, 65 \%)$ as a foam. $[\alpha]_{\mathrm{D}}{ }^{20}$ 16.2.; ${ }^{1} \mathrm{H}$ NMR $\delta 7.64-7.61$ (m, 4H, Ar), $7.35-7.18\left(\mathrm{~m}, 36 \mathrm{H}\right.$, aryl-H), $6.08\left(\mathrm{~d}, 1 \mathrm{H}, J_{1,2}=3.6 \mathrm{~Hz}, \mathrm{H}-1\right)$, $4.86-4.38\left(\mathrm{~m}, 12 \mathrm{H}, \mathrm{PhCH}_{2}\right), 4.54\left(\mathrm{~d}, 1 \mathrm{H}, J_{5,4}=9.9 \mathrm{~Hz}, \mathrm{H}-5\right)$, $4.48\left(\mathrm{~d}, 1 \mathrm{H}, J_{3,4}=7.5 \mathrm{~Hz}, \mathrm{H}-3^{\prime}\right), 4.45\left(\mathrm{dd}, 1 \mathrm{H}, J_{4,3}=7.5, J_{4,5}=\right.$ $\left.15.0 \mathrm{~Hz}, \mathrm{H}-4^{\prime}\right), 4.03$ (dd, $1 \mathrm{H}, J_{6,5}=3.8, J_{6,6}, 11.5 \mathrm{~Hz}, \mathrm{H}-6^{\prime}$ ), 3.94 (m, 2H, H-3, H-5'), 3.85 (dd, $1 \mathrm{H}, J_{6,5}=4.1 \mathrm{~Hz}, J_{6,6},=11.5$ Hz, H-6'), 3.71 (dd, $\left.1 \mathrm{H}, J_{4,3}=9.1 \mathrm{~Hz}, J_{4,5}=9.9 \mathrm{~Hz}, \mathrm{H}-4\right), 3.67$ $\left(\mathrm{d}, 1 \mathrm{H}, J_{\mathrm{gem}}=10.8 \mathrm{~Hz}, \mathrm{H}-1^{\prime}\right), 3.62\left(\mathrm{~d}, 3 \mathrm{H}, J_{\mathrm{H}, \mathrm{P}}=11.1 \mathrm{~Hz}\right.$, $\left.\mathrm{OCH}_{3}\right), 3.61\left(\mathrm{~d}, 3 \mathrm{H}, J_{\mathrm{H}, \mathrm{P}}=11.2 \mathrm{~Hz}, \mathrm{OCH}_{3}\right), 3.56\left(\mathrm{~d}, 1 \mathrm{H}, J_{\mathrm{gem}}\right.$ $=10.8 \mathrm{~Hz}, \mathrm{H}-1$ '), $3.43\left(\mathrm{dd}, 1 \mathrm{H}, J_{2,1}=3.6, J_{2,3}=9.7 \mathrm{~Hz}, \mathrm{H}-2\right)$, $3.26\left(\mathrm{dd}, 1 \mathrm{H}, J_{7,7^{\prime}}=15.7 \mathrm{~Hz}, J_{\mathrm{H}, \mathrm{P}}=20.3 \mathrm{~Hz}, \mathrm{H}-7\right), 2.97(\mathrm{dd}$, $\left.1 \mathrm{H}, J_{7,7^{\prime}}=15.7 \mathrm{~Hz}, J_{\mathrm{H}, \mathrm{P}}=20.8 \mathrm{~Hz}, \mathrm{H}-7\right), 1.06(\mathrm{~s}, 9 \mathrm{H}, t-\mathrm{Bu})$; ${ }^{13} \mathrm{C}$ NMR $\delta 198.5(\mathrm{~d}, J 7.4 \mathrm{~Hz}, \mathrm{C}=\mathrm{O}), 138.7,138.5,138.2$, 138.1, 137.8, 137.7, 135.6, 135.4, 133.1, 132.7, 129.8, 129.8, 128.6-127.5 (Ar), 104.5 (C-2'), 89.2 (C-1), 83.3 (C-3'), 81.5 (C-3), 80.8 (C-4'), 80.5 (C-5'), 79.5 (C-2), 77.9 (C-4), 75.7 $\left(\mathrm{PhCH}_{2}\right), 74.9$ (d, J 5.0 Hz, C-5), $74.8\left(\mathrm{PhCH}_{2}\right), 73.5\left(\mathrm{PhCH}_{2}\right)$, $73.4\left(\mathrm{PhCH}_{2}\right), 72.9\left(\mathrm{PhCH}_{2}\right), 72.0\left(\mathrm{PhCH}_{2}\right), 72.0(\mathrm{C}-1 '), 63.5$ 
(C-6'), $52.8\left(\mathrm{~d}, J=6.3 \mathrm{~Hz}, \mathrm{OCH}_{3}\right), 52.6\left(\mathrm{~d}, J=6.4 \mathrm{~Hz}, \mathrm{OCH}_{3}\right)$, 37.9 (d, $J=136.9 \mathrm{~Hz}, \mathrm{C}-7), 26.9\left(t-\mathrm{Bu}-\mathrm{CH}_{3}\right), 19.2(t-\mathrm{Bu}-\mathrm{C}) ;{ }^{31} \mathrm{P}$ NMR $\left(\mathrm{CDCl}_{3}\right) \delta 23.5$; anal. calcd for $\mathrm{C}_{73} \mathrm{H}_{81} \mathrm{O}_{14} \mathrm{PSi}$ (1241.51): C, 70.62; H, 6.58; found: C, 70.44; H, 6.79.

Synthesis of ketone 10. To a solution of aldehyde 7 (200 mg, $0.18 \mathrm{mmol})$, phosphonate $9(220 \mathrm{mg}, 0.18 \mathrm{mmol})$, and 18-crown-6 $(70 \mathrm{mg})$ in toluene $(25 \mathrm{~mL})$ potassium carbonate (300 mg) was added and the suspension was stirred at $\mathrm{rt}$ for 4 days. The solvents were evaporated and the residue was purified by column chromatography (hexane-ethyl acetate, $40: 1 \rightarrow$ $5: 1)$ to afford title compound $\mathbf{1 0}$ as a foam $(287 \mathrm{mg} ; 73 \%)$. $[\alpha]_{\mathrm{D}}{ }^{20} 41.8 ;{ }^{1} \mathrm{H}$ NMR $\delta$ 7.62-7.61 (m, 8H, Ar), 7.28-7.06 (m, $73 \mathrm{H}, \mathrm{Ar},=C \mathrm{H}-\mathrm{CO}), 6.75(\mathrm{dd}, 1 \mathrm{H}, J=1.8 \mathrm{~Hz}, 15.7 \mathrm{~Hz},=\mathrm{CH})$, 5.90 (d, 1H, $J=3.5 \mathrm{~Hz}, \mathrm{H}-1 \mathrm{~A}), 5.82$ (d, $1 \mathrm{H}, J=3.5 \mathrm{~Hz}, \mathrm{H}-1 \mathrm{~B})$, 4.74-4.26(m, 24H, $\left.12 \times \mathrm{PhCH}_{2}\right), 4.67(\mathrm{~d}, 1 \mathrm{H}, J=10.2 \mathrm{~Hz}$, $\mathrm{H}-5 \mathrm{~A}), 4.58$ (d, 1H, $J=12.3 \mathrm{~Hz}, \mathrm{H}-5 \mathrm{~B}), 4.37-4.26$ (m, 4H, furanose), 4.00-3.80 (m, 8H, H-3A, H-3B, $4 \times$ H-6', $2 \times$ furanose), 3.77 (d, 1H, $J=11.0 \mathrm{~Hz}, \mathrm{H}-1$ 'B), 3.68 (d, 1H, $J=10.9$ Hz, H-1'A), 3.50 (dd, 1H, $J=9.1 \mathrm{~Hz}, 10.0 \mathrm{~Hz}, \mathrm{H}-4 \mathrm{~A}$ ), 3.46 (d, $1 \mathrm{H}, J=10.9 \mathrm{~Hz}, \mathrm{H}-1$ 'A), 3.42-3.38 (m, 2H, H-1'B, H-2A), 3.25 $(\mathrm{dd}, 1 \mathrm{H}, J=3.5 \mathrm{~Hz}, 9.6 \mathrm{~Hz}, \mathrm{H}-2 \mathrm{~A}), 2.97(\mathrm{dd}, 1 \mathrm{H}, J=9.1 \mathrm{~Hz}$, $9.9 \mathrm{~Hz}, \mathrm{H}-4 \mathrm{~B}), 1.03$ (s, 9H, $t$-Bu), 1.02 (s, 9H, $t$-Bu); ${ }^{13} \mathrm{C}$ NMR $\delta 195.2(\mathrm{C}=\mathrm{O}), 144.7(=\mathrm{CH}-\mathrm{CO}), 138.9,138.8,138.4,138.3$, $138.2,138.1,138.1,138.0,137.9,137.9,137.8,137.7,135.6$, $135.6,135.5,133.4,133.3,133.2,133.1,129.7,129.7,129.7$, 129.6, 128.4-127.4 (Ar), $124.8(=C H) 105.1\left(\mathrm{C}-2^{\prime} \mathrm{A}\right), 104.8$ (C-2'B), 90.3 (C-1A), 90.2 (C-1B), 84.4, 83.9, 83.3, 83.2, 81.8 (C-4B), 81.7, 81.7, $81.5(\mathrm{C}-3 \mathrm{~A}), 81.4(\mathrm{C}-3 \mathrm{~B}), 79.8$ (C-2A, C-2B), $79.6(\mathrm{C}-4 \mathrm{~A}), 75.7\left(\mathrm{PhCH}_{2}\right), 75.4\left(\mathrm{PhCH}_{2}\right), 74.9(\mathrm{C}-5 \mathrm{~A}$, $\left.\mathrm{PhCH}_{2}\right), 74.4\left(\mathrm{PhCH}_{2}\right), 73.5\left(\mathrm{PhCH}_{2}\right), 73.4\left(\mathrm{PhCH}_{2}\right), 73.1$ $\left(\mathrm{PhCH}_{2}\right), 73.0\left(\mathrm{PhCH}_{2}\right), 72.6\left(\mathrm{PhCH}_{2}\right), 72.4\left(\mathrm{PhCH}_{2}\right), 72.1$ $\left(\mathrm{PhCH}_{2}\right), 71.8\left(\mathrm{PhCH}_{2}\right), 70.6\left(\mathrm{C}-1^{\prime}\right), 70.4\left(\mathrm{C}-1^{\prime}\right), 69.8(\mathrm{C}-5 \mathrm{~B})$, $65.3\left(\mathrm{C}-6\right.$ '), $65.0\left(\mathrm{C}^{-6}\right), 27.0(t-\mathrm{Bu}), 27.0\left(t\right.$-Bu), $19.3\left(\mathrm{CH}_{3}\right)$, $19.3\left(\mathrm{CH}_{3}\right)$; anal. calcd for $\mathrm{C}_{141} \mathrm{H}_{148} \mathrm{O}_{21} \mathrm{Si}_{2}$ (2234.91): $\mathrm{C}$, 75.78; H, 6.68; found: C, 75.59; H, 6.80.

Stereoselective reduction of ketone $\mathbf{1 0}$. To an ice-cooled solution of ketone $10(270 \mathrm{mg}, 0.12 \mathrm{mmol})$ in $\mathrm{Et}_{2} \mathrm{O}(15 \mathrm{~mL})$, an etheral solution of zinc borohydride $(0.6 \mathrm{mmol})$ was added and the mixture was stirred at $0{ }^{\circ} \mathrm{C}$ for $1 \mathrm{~h}$. Water ( 10 drops) was added to decompose excess of hydride, the solvents were evaporated, and the residue was purified by column chromatography (hexane-ethyl acetate, 10:1 $\rightarrow 5: 1$ ) to afford title compound $11(176 \mathrm{mg}, 65 \%)$ as a foam. $[\alpha]_{\mathrm{D}}{ }^{20} 40.4 ;{ }^{1} \mathrm{H}$ NMR $\delta$ 7.63-7.61 (m, 8H, Ar), 7.30-7.05 (m, 72H, Ar), 5.97 (dd, 1H, $J$ $=7.9 \mathrm{~Hz}, 15.6 \mathrm{~Hz},=\mathrm{CH}-\mathrm{CHOH}), 5.83(\mathrm{~d}, 2 \mathrm{H}, J=3.6 \mathrm{~Hz}$, $\mathrm{H}-1 \mathrm{~A}, \mathrm{H}-1 \mathrm{~B}), 5.76(\mathrm{dd}, 1 \mathrm{H}, J=5.5 \mathrm{~Hz}, 15.6 \mathrm{~Hz},=\mathrm{C} H)$, 4.80-4.73 (m, 5H, $\left.\mathrm{PhCH}_{2}\right), 4.63-4.41\left(\mathrm{~m}, 17 \mathrm{H}, \mathrm{PhCH}_{2}, \mathrm{H}-3\right.$ ', H-5B), 4.39-4.34 (m, 6H, PhCH 2 , H-3', H-3', H-4', H-6A), $4.32-4.28$ (m, 1H, H-4'), 4.25 (dd, $1 \mathrm{H}, J=2.2 \mathrm{~Hz}, 10.3 \mathrm{~Hz}$,
H-5A), 4.13 (d, 1H, $\left.J=10.9 \mathrm{~Hz}, \mathrm{PhCH}_{2}\right), 4.06-4.04(\mathrm{~m}, 1 \mathrm{H}$, H-5'), 3.99-3.95 (m, 2H, $2 \times \mathrm{H}-6$ '), 3.92-3.89 (m, 2H, H-5', H-6'), 3.86-3.79 (m, 3H, H-3A, H-1', H-3B), 3.65 (d, 1H, $J=$ $\left.11.0 \mathrm{~Hz}, \mathrm{H}-1^{\prime}\right), 3.52$ (d, 1H, $\left.J=11.0 \mathrm{~Hz}, \mathrm{H}-1^{\prime}\right), 3.49$ (d, 1H, H-1'), 3.35 (dd, 1H, $J=3.5 \mathrm{~Hz}, 9.6 \mathrm{~Hz}, \mathrm{H}-2 \mathrm{~B}), 3.27-3.24$ (m, $1 \mathrm{H}, \mathrm{H}-4 \mathrm{~A}), 3.21-3.16$ (m, 2H, H-2A, H-4B), 1.03 (s, 9H, $t$-Bu), $1.02(\mathrm{~s}, 9 \mathrm{H}, t-\mathrm{Bu}) ;{ }^{13} \mathrm{C} \mathrm{NMR} \delta 139.0,138.9,138.6,138.6$, $138.5,138.4,138.3,138.1,138.0,137.9,137.8,137.7,135.7$, $135.6,135.5,135.5,133.5,133.3,133.3,132.8,131.3(=\mathrm{CH})$, 130.4 (=CH-CHOH), 129.8, 129.7, 129.7, 129.6, 128.5-127.3 (Ar), 105.0 (C-2'), 104.5 (C-2'), 90.2 (C-1B), 89.0 (C-1A), 84.5 (C-3'), 83.6 (C-4'), 83.1 (C-4'), 82.3 (C-3A), 82.2 (C-4B), 81.8 (C-5'), $81.6\left(\mathrm{C}^{-3}\right), 81.5$ (C-3B), $81.0\left(\mathrm{C}-5^{\prime}\right), 80.4(\mathrm{C}-2 \mathrm{~A}), 80.0$ (C-2B), $78.4(\mathrm{C}-4 \mathrm{~A}), 75.5\left(\mathrm{PhCH}_{2}\right), 75.4\left(\mathrm{PhCH}_{2}\right), 74.6$ $\left(\mathrm{PhCH}_{2}\right), 74.4\left(\mathrm{PhCH}_{2}\right), 73.6\left(\mathrm{PhCH}_{2}\right), 73.4\left(\mathrm{PhCH}_{2}\right), 73.4$ (C-5A), $73.1\left(\mathrm{PhCH}_{2}\right), 73.0\left(\mathrm{PhCH}_{2}\right), 72.7\left(\mathrm{PhCH}_{2}\right), 72.6$ $\left(\mathrm{PhCH}_{2}\right), 71.8\left(\mathrm{PhCH}_{2}\right), 71.7\left(\mathrm{PhCH}_{2}\right), 71.5(\mathrm{C}-6 \mathrm{~A}), 71.4$ (C-1'), 70.5 (C-1'), 70.0 (C-5B), 65.8 (C-6'), 63.7 (C-6'), 27.0 $(t-\mathrm{Bu}), 27.0(t-\mathrm{Bu}), 19.3\left(\mathrm{CH}_{3}\right), 19.3\left(\mathrm{CH}_{3}\right)$; anal. calcd for $\mathrm{C}_{141} \mathrm{H}_{150} \mathrm{O}_{21} \mathrm{Si}_{2}$ (2236.92): C, 75.71; H, 6.76; found: C, 75.51; $\mathrm{H}, 6.63$.

Benzylation of 11. To a solution of alcohol 11 (82 $\mathrm{mg}$, $0.037 \mathrm{mmol})$ in DMF $(1 \mathrm{~mL})$, sodium hydride $(60 \%$ suspension in mineral oil, $7 \mathrm{mg}$ ) was added and the mixture was stirred for $30 \mathrm{~min}$ at rt. Benzyl bromide (11 $\mu \mathrm{L}, 0.092 \mathrm{mmol})$ was added, and stirring was continued overnight. Excess of sodium hydride was decomposed with methanol $(0.5 \mathrm{~mL})$. The product was isolated by column chromatography (hexane-ethyl acetate, 10:1 $\rightarrow 5: 1)$ to afford $12(34 \mathrm{mg}, 40 \%)$ as a foam. $[\alpha]_{\mathrm{D}}{ }^{20} 21.8(c 0.9$, $\left.\mathrm{CH}_{2} \mathrm{Cl}_{2}\right) ;{ }^{1} \mathrm{H}$ NMR $\delta$ 7.67-7.63 (m, 8H, Ar), 7.28-7.00 (m, $77 \mathrm{H}, \mathrm{Ar}), 5.93-5.89(\mathrm{~m}, 2 \mathrm{H}, \mathrm{H}-1 \mathrm{~B},=\mathrm{CH}-\mathrm{CHOH}), 5.63-5.59$ $(\mathrm{m}, 2 \mathrm{H}, \mathrm{H}-1 \mathrm{~A},=\mathrm{CH}), 4.79-4.50\left(\mathrm{~m}, 13 \mathrm{H}, \mathrm{H}-5 \mathrm{~B}, \mathrm{PhCH}_{2}\right)$, 4.47-4.16 (m, 18H, H-5A, PhCH $)$, 4.10-3.75 (m, 12H, H-6A, $4 \times \mathrm{H}^{-6}, \mathrm{H}-3 \mathrm{~B}, 2 \times \mathrm{H}-1^{\prime}, \mathrm{H}-3 \mathrm{~A}, 2 \times$ furanose, $\left.\mathrm{PhCH}\right)$, 3.56-3.52 (m, 2H, $\left.2 \times \mathrm{H}-1^{\prime}\right), 3.41(\mathrm{dd}, 1 \mathrm{H}, J=3.6,9.7 \mathrm{~Hz}$, H-2B), 3.19-3.14 (m, 2H, H-4A, H-4B), 2.92 (dd, 1H, $J=3.5$, $9.6 \mathrm{~Hz}, \mathrm{H}-2 \mathrm{~A}), 1.03$ (s, 9H, $t$-Bu), 1.03 (s, 9H, $t$-Bu); ${ }^{13} \mathrm{C} \mathrm{NMR}$ $\delta: 139.0,138.8,138.7,138.6,138.5,138.4,138.3,138.3,138.3$, $138.3,138.2,138.0,137.8,135.6,135.5,135.5,135.5,134.4$ $(=C \mathrm{H}), 133.7,133.6,133.4,133.2,129.7,129.6,129.6,129.6$, 128.3-127.0 (Ar), 104.4 (C-2'), 104.4 (C-2'), 90.1 (C-1A), 89.9 (C-1B), 84.5, 84.1, 83.2, 82.2, 82.0 (C-3A), 82.0 (C-4B), 81.6 (C-3B, C-6A), 80.4 (C-2A), 79.9 (C-2B), 78.6, 78.2 (C-4A), $75.6\left(\mathrm{PhCH}_{2}\right), 75.2\left(\mathrm{PhCH}_{2}\right), 74.6\left(\mathrm{PhCH}_{2}\right), 74.2\left(\mathrm{PhCH}_{2}\right)$, $73.6\left(\mathrm{PhCH}_{2}\right), 73.2\left(\mathrm{PhCH}_{2}\right), 73.1\left(\mathrm{PhCH}_{2}\right), 73.0\left(\mathrm{PhCH}_{2}\right)$, $72.7\left(\mathrm{PhCH}_{2}\right), 72.6(\mathrm{C}-5 \mathrm{~A}), 72.5\left(\mathrm{PhCH}_{2}\right), 71.9\left(\mathrm{PhCH}_{2}\right), 71.8$ $\left(\mathrm{PhCH}_{2}\right), 71.0\left(\mathrm{C}^{\prime} 1^{\prime}\right), 70.3,70.2\left(\mathrm{C}-1^{\prime}\right), 69.9,66.7$ (C-6'), 65.6 $\left(\mathrm{C}-6{ }^{\prime}\right), 27.0(t-\mathrm{Bu}), 26.9(t-\mathrm{Bu}), 19.3\left(\mathrm{CH}_{3}\right), 19.3\left(\mathrm{CH}_{3}\right)$; anal. calcd for $\mathrm{C}_{148} \mathrm{H}_{156} \mathrm{O}_{21} \mathrm{Si}_{2}$ (2327.05): C, 76.39; H, 6.76; found: C, 76.44; H, 6.73 . 
Dihydroxylation of the double bond of 12 . Olefin $12(60 \mathrm{mg}$, $0.026 \mathrm{mmol})$ and $\mathrm{OsO}_{4}(30 \mathrm{mg}, 0.120 \mathrm{mmol})$ were dissolved in pyridine $(4 \mathrm{~mL})$, and stirred for $48 \mathrm{~h}$. The solvent was evaporated, the residue was dissolved in ethyl acetate $(10 \mathrm{~mL})$, to which sat. aq $\mathrm{Na}_{2} \mathrm{~S}_{2} \mathrm{O}_{3}(1 \mathrm{~mL})$ was added, and the suspension was stirred for 2 days. The organic layer was separated, solvents were evaporated to dryness and the residue was purified by column chromatography (hexane-ethyl acetate, $5: 1$ ) to afford $13(44 \mathrm{mg}, 73 \%)$ as colorless glass. $[\alpha]_{\mathrm{D}}{ }^{20} 22.8 ;{ }^{1} \mathrm{H}$ NMR $\delta$ 7.64-7.59 (m, 8H, Ar), 7.27-6.98 (m, 77H, Ar), 5.83 (bs, 1H, H-1A), 5.80 (d, 1H, $\left.J_{1,2}=3.5 \mathrm{~Hz}, \mathrm{H}-1 \mathrm{~B}\right), 4.82-4.69$ (m, 5H, $\left.\mathrm{PhCH}_{2}\right), 4.65-4.49\left(\mathrm{~m}, 11 \mathrm{H}, \mathrm{PhCH}_{2}\right.$, sugar-H), 4.45-4.34 (m, $10 \mathrm{H}, \mathrm{PhCH}_{2}, 2 \times$ sugar-H), 4.29-4.23 (m, 7H, $\mathrm{PhCH}_{2}, 5 \times$ sugar-H), 4.08-4.05 (m, 2H, $\mathrm{PhCH}_{2}$, sugar-H), 4.01-3.95 (m, $6 \mathrm{H}, 3 \times \mathrm{H}-6$ ', $3 \times$ sugar-H), 3.89-3.78 (m, 4H, H-1', H-3A, H-3B, H-6'), 3.67 (d, 1H, J=11.1 Hz, H-1'), 3.58-3.55 (m, 2H, H-1', H-1'), 3.37 (dd, 1H, J=3.6 Hz, 9.6 Hz, H-2B), 3.36-3.32 (m, 2H, H-2A, sugar-H), $1.02(\mathrm{~s}, 9 \mathrm{H}, t-\mathrm{Bu}), 1.01(\mathrm{~s}, 9 \mathrm{H}, t-\mathrm{Bu})$; ${ }^{13} \mathrm{C}$ NMR $\delta 139.1,139.0,138.9,138.8,138.6,138.3,138.2$, $138.2,138.0,137.9,137.7,137.6,135.6,135.6,135.5,135.5$, $133.6,133.4,133.3,132.9,129.7,129.7,129.6,129.5,128.4-$ 127.1 (Ar), 105.1 (C-2'), 104.7 (C-2'), 90.7 (C-1B), 89.4 (C-1A), 84.4, 83.5, 82.5 (C-3B), 82.0, 81.9, 81.6 (C-3A), 81.2, 80.4 (C-2B), 79.7 (C-2A), 79.3, 78.6, $75.5\left(\mathrm{PhCH}_{2}\right), 75.3$ $\left(\mathrm{PhCH}_{2}\right), 74.6\left(\mathrm{PhCH}_{2}\right), 74.3,74.3\left(\mathrm{PhCH}_{2}\right), 73.5\left(\mathrm{PhCH}_{2}\right)$, $73.2\left(\mathrm{PhCH}_{2}\right), 73.1\left(\mathrm{PhCH}_{2}\right), 73.1\left(\mathrm{PhCH}_{2}\right), 72.7\left(\mathrm{PhCH}_{2}\right)$, $\left.72.5\left(\mathrm{PhCH}_{2}\right), 71.9\left(\mathrm{PhCH}_{2}\right), 71.8\left(\mathrm{PhCH}_{2}\right), 70.9(\mathrm{C}-1)\right), 70.7$, 70.1 (C-1'), 69.7, 68.2, 66.4 (C-6'), 64.2 (C-6'), $27.0(t-\mathrm{Bu}), 19.3$ $\left(\mathrm{CH}_{3}\right), 19.2\left(\mathrm{CH}_{3}\right)$; MS (ESI): $2383.08[\mathrm{M}+\mathrm{Na}]^{+}$; anal. calcd for $\mathrm{C}_{148} \mathrm{H}_{158} \mathrm{O}_{23} \mathrm{Si}_{2}$ (2361.06): C, 75.29; $\mathrm{H}, 6.75$; found: $\mathrm{C}$, $75.29 ; \mathrm{H}, 6.79$.

Synthesis of olefin 15. To a suspension of methyltriphenylphosphonium bromide ( $895 \mathrm{mg}, 2.50 \mathrm{mmol})$ in benzene $(20 \mathrm{~mL})$ a $2.5 \mathrm{M}$ solution of BuLi in hexane $(0.95 \mathrm{~mL}$, $2.30 \mathrm{mmol}$ ) was added and the mixture was stirred at $\mathrm{rt}$ for $90 \mathrm{~min}$. A solution of aldehyde $7(298 \mathrm{mg}, 0.27 \mathrm{mmol})$ in benzene $(5 \mathrm{~mL})$ was added, the mixture was stirred for another $45 \mathrm{~min}$, quenched with water $(100 \mu \mathrm{L})$, concentrated, and the residue was purified by column chromatography (hexane-ethyl acetate, $15: 1 \rightarrow 10: 1)$ to afford $\mathbf{1 5}(172 \mathrm{mg}, 58 \%)$ as a foam. $[\alpha]_{\mathrm{D}}{ }^{20} 24.0 ;{ }^{1} \mathrm{H}$ NMR $\delta$ 7.67-7.64 (m, 4H, Ar), 7.35-7.18 (m, $36 \mathrm{H}, \mathrm{Ar}), 5.77-5.72$ (m, 2H, H-1,6), 5.12-5.09 (m, 1H, H-7), 5.02-5.00 (m, 1H, H-7), 4.82-4.50 (m, 11H, $\left.\mathrm{PhCH}_{2}\right), 4.45-4.43$ (m, 3H, H-3',5,PhCH), 4.30 (t, $\left.1 \mathrm{H}, J_{4,3}=J_{4,5}=7.4 \mathrm{~Hz}, \mathrm{H}-4^{\prime}\right)$, 4.05-4.02 (m, 1H, H-5'), $3.94\left(\mathrm{dd}, 1 \mathrm{H}, J_{6,5}=4.8 \mathrm{~Hz}, J_{6,6}\right.$ ' = $\left.11.1 \mathrm{~Hz}, \mathrm{H}-6^{\prime}\right), 3.90-3.87$ (m, 2H, H-3,6'), 3.69 (d, 1H, $J_{\text {gem }}=$ $\left.11.0 \mathrm{~Hz}, \mathrm{H}-1^{\prime}\right), 3.50$ (d, 1H, $\left.J_{\text {gem }}=11.0 \mathrm{~Hz}, \mathrm{H}-1^{\prime}\right), 3.44(\mathrm{dd}, 1 \mathrm{H}$, $\left.J_{2,1}=3.7 \mathrm{~Hz}, J_{2,3}=9.7 \mathrm{~Hz}, \mathrm{H}-2\right), 3.16\left(\mathrm{dd}, 1 \mathrm{H}, J_{4,3}=9.4 \mathrm{~Hz}\right.$, $\left.J_{4,5}=9.5 \mathrm{~Hz}, \mathrm{H}-4\right), 1.06(\mathrm{~s}, 9 \mathrm{H}, t-\mathrm{Bu}) ;{ }^{13} \mathrm{C} \mathrm{NMR} \delta 138.9$, 138.6, 138.4, 138.3, 138.0, 135.7, 135.6, 135.6, 133.5, 133.3,
129.6, 129.6, 128.3-127.5 (Ar), 117.0 (C-7), 104.3 (C-2'), 89.2 (C-1), 83.8 (C-3'), 82.5 (C-4), 82.3 (C-4'), 81.6 (C-3), 81.2 (C-5'), 80.0 (C-2), $75.6\left(\mathrm{PhCH}_{2}\right), 74.8\left(\mathrm{PhCH}_{2}\right), 73.4\left(\mathrm{PhCH}_{2}\right)$, $72.9\left(\mathrm{PhCH}_{2}\right), 72.6\left(\mathrm{PhCH}_{2}\right), 72.3\left(\mathrm{PhCH}_{2}\right), 71.4(\mathrm{C}-1 ', 5), 64.9$ (C-6'), $26.9\left(t-\mathrm{Bu}-\mathrm{CH}_{3}\right), 19.3(t-\mathrm{Bu}-C)$; anal. calcd for $\mathrm{C}_{71} \mathrm{H}_{76} \mathrm{O}_{10} \mathrm{Si}$ (1117.47): C, 76.31; H, 6.86; found: $\mathrm{C}, 76.42 ; \mathrm{H}$, 6.99 .

\section{Ozonolytic cleavage of the double bond in sucrose dimer 11.} Determination of the configuration at the carbinol center. Ozone was passed through a cooled solution of $\mathbf{1 1}(51 \mathrm{mg}$, $0.023 \mathrm{mmol})$ in $\mathrm{CH}_{2} \mathrm{Cl}_{2}(10 \mathrm{~mL})$ until the blue color persisted (10 min). Dimethyl disulfide $(210 \mu \mathrm{L})$ was added, the mixture was stirred for $10 \mathrm{~min}$, concentrated, and the residue was dissolved in methanol $(10 \mathrm{~mL})$. Sodium borohydride $(40 \mathrm{mg})$ was added, the mixture was stirred for $1 \mathrm{~h}$, concentrated, and the crude product was purified by column chromatography (hexane-ethyl acetate, 7:3) to afford sucrose 6 (16 mg, 63\%) and diol 14 (18 mg, 69\%), both as foam.

Synthesis of diols 14 and 16. To a solution of 15 (145 mg, $0.13 \mathrm{mmol})$ in THF $(10 \mathrm{~mL})$, tert-butyl alcohol $(500 \mu \mathrm{L})$, water $(50 \mu \mathrm{L})$, NMO $(80 \mathrm{mg}, 0.68 \mathrm{mmol})$, and $\mathrm{OsO}_{4}(8 \mathrm{wt} \%$ in $t$-BuOH, $150 \mu \mathrm{L}, 0.035 \mathrm{mmol})$ were added, and the mixture was stirred at $\mathrm{rt}$ for $18 \mathrm{~h}$. Saturated aq $\mathrm{Na}_{2} \mathrm{~S}_{2} \mathrm{O}_{3}(0.2 \mathrm{~mL})$ was added, the mixture was stirred for $1 \mathrm{~h}$ at $\mathrm{rt}$, concentrated, and the products were isolated by column chromatography (hexane-ethyl acetate, $5: 1 \rightarrow 3: 1)$ to afford of 14 (60 mg; 40\%) and $16(58 \mathrm{mg}$ $39 \%)$, both as foam.

Data for 14: $[\alpha]_{\mathrm{D}}{ }^{20} 32.6 ;{ }^{1} \mathrm{H}$ NMR $\delta 7.64-7.62(\mathrm{~m}, 4 \mathrm{H}$, aryl-H), 7.36-7.14 (m, 36H, Ar), 6.04 (d, 1H, $\left.J_{1,2}=3.8 \mathrm{~Hz}, \mathrm{H}-1\right)$, 4.95-4.39 (m, 10H, $\left.\mathrm{PhCH}_{2}\right), 4.47-4.45$ (m, 4H, H-3',4', $\mathrm{PhCH}_{2}$ ), $4.14\left(\mathrm{dd}, 1 \mathrm{H}, J_{5,4}=10.2, J_{5,6}=4.1 \mathrm{~Hz}, \mathrm{H}-5\right), 4.03\left(\mathrm{dd}, 1 \mathrm{H}, J_{6,5}\right.$ $\left.=3.4, J_{6,6},=11.6 \mathrm{~Hz}, \mathrm{H}-6^{\prime}\right), 3.93-3.89$ (m, 2H, H-3,5'), 3.84-3.79 (m, 2H, H-6,6'), 3.65-3.62 (m, 2H, H-1',7), 3.55 (d, $\left.1 \mathrm{H}, J_{\mathrm{gem}}=10.8 \mathrm{~Hz}, \mathrm{H}-1^{\prime}\right), 3.49-3.43(\mathrm{~m}, 3 \mathrm{H}, \mathrm{H}-2,4,7), 1.07(\mathrm{~s}$, $9 \mathrm{H}, t-\mathrm{Bu}) ;{ }^{13} \mathrm{C}$ NMR $\delta 138.6,138.2,138.0,137.9,137.8,137.3$, 135.7, 135.4, 133.0, 132.6, 129.8, 129.7, 128.7-127.6 (Ar), 104.3 (C-2'), 88.2 (C-1), 82.9 (C-3'), 82.0 (C-3), 80.3 (C-5'), 80.1 (C-2,4'), $79.0(\mathrm{C}-4), 75.5\left(\mathrm{PhCH}_{2}\right), 74.7\left(\mathrm{PhCH}_{2}\right), 73.4$ $\left(\mathrm{PhCH}_{2}\right), 73.1\left(\mathrm{PhCH}_{2}\right), 72.8\left(\mathrm{PhCH}_{2}\right), 72.3(\mathrm{C}-1 '), 72.2(\mathrm{C}-6)$, $71.9\left(\mathrm{PhCH}_{2}\right), 71.3$ (C-5), $63.0(\mathrm{C}-6)$ ), $62.9(\mathrm{C}-7), 26.9(t-\mathrm{Bu}-$ $\mathrm{CH}_{3}$ ), 19.2 ( $t$-Bu- $C$ ); anal. calcd for $\mathrm{C}_{71} \mathrm{H}_{78} \mathrm{O}_{12} \mathrm{Si}$ (1151.49): $\mathrm{C}$, 74.06; H, 6.83; found: C, 74.00; H, 6.79.

Data for 16: $[\alpha]_{\mathrm{D}}{ }^{20} 21.6$; ${ }^{1} \mathrm{H}$ NMR $\delta 7.64-7.62(\mathrm{~m}, 4 \mathrm{H}, \mathrm{Ar})$, $7.37-7.16\left(\mathrm{~m}, 36 \mathrm{H}\right.$, aryl-H), $6.04\left(\mathrm{~d}, 1 \mathrm{H}, J_{1,2}=3.6 \mathrm{~Hz}, \mathrm{H}-1\right)$, 4.93-4.40 (m, 12H, $\left.\mathrm{PhCH}_{2}\right), 4.40$ (d, 1H, J = 6.5 Hz, H-3'), $4.32\left(\mathrm{t}, 1 \mathrm{H}, J_{4,3}=J_{4,5}=6.7 \mathrm{~Hz}, \mathrm{H}-4\right), 4.03-3.99(\mathrm{~m}, 2 \mathrm{H}$, H-5,6'), $3.95\left(\mathrm{t}, 1 \mathrm{H}, J_{3,2}=J_{3,4}=9.4 \mathrm{~Hz}, \mathrm{H}-3\right), 3.92-3.91(\mathrm{~m}$, 
$\left.1 \mathrm{H}, \mathrm{H}-5^{\prime}\right), 3.81$ (dd, $\left.1 \mathrm{H}, J_{6,5}=4.0 \mathrm{~Hz}, J_{6,6}{ }^{\prime}=11.4 \mathrm{~Hz}, \mathrm{H}-6^{\prime}\right)$, 3.74-3.72 (m, 1H, H-6), 3.70-3.66 (m, 2H, H-1',4), 3.55 (d, 1H, $\left.J_{\text {gem }}=10.9 \mathrm{~Hz}, \mathrm{H}-1^{\prime}\right), 3.48-3.42(\mathrm{~m}, 3 \mathrm{H}, \mathrm{H}-2,7,7), 1.07$ (s, 9H, $t$-Bu); ${ }^{13} \mathrm{C}$ NMR $\delta 138.8,138.5,138.3,137.9,137.7,137.5$, $135.7,135.5,132.9,132.7,129.8,129.8,128.4-127.5$ (Ar), 104.8 (C-2'), 89.3 (C-1), 83.2 (C-3'), 81.4 (C-3), 81.1 (C-4'), $80.8\left(\mathrm{C}-5\right.$ '), 79.9 (C-2), $77.3(\mathrm{C}-4), 75.5\left(\mathrm{PhCH}_{2}\right), 75.1$ $\left(\mathrm{PhCH}_{2}\right), 73.5\left(\mathrm{PhCH}_{2}\right), 73.2\left(\mathrm{PhCH}_{2}\right), 72.5\left(\mathrm{PhCH}_{2}\right), 72.0$ $\left(\mathrm{PhCH}_{2}\right), 71.9$ (C-5), $71.6\left(\mathrm{C}-1^{\prime}\right), 68.9$ (C-6), 64.5 (C-7), 63.1 $\left(\mathrm{C}-6\right.$ '), $26.9\left(t-\mathrm{Bu}-\mathrm{CH}_{3}\right), 19.2(t-\mathrm{Bu}-\mathrm{C})$; anal. calcd for $\mathrm{C}_{71} \mathrm{H}_{78} \mathrm{O}_{12} \mathrm{Si}$ (1151.49): C, 74.06; H, 6.83; found: C, 73.94; $\mathrm{H}$, 6.66 .

Both compounds were further characterized as diacetates: 14-Ac and 16-Ac.

Data for 14-Ac: $[\alpha]_{\mathrm{D}}{ }^{20} 33.3 ;{ }^{1} \mathrm{H}$ NMR $\delta$ 7.66-7.64 (m, 4H, Ar), $7.34-7.21\left(\mathrm{~m}, 36 \mathrm{H}\right.$, aryl-H), $5.82\left(\mathrm{~d}, 1 \mathrm{H}, J_{1,2}=3.6 \mathrm{~Hz}, \mathrm{H}-1\right)$, 5.48 (m, 1H, H-6), 4.93-4.55 (m, 10H, $\left.\mathrm{PhCH}_{2}\right), 4.46-4.42(\mathrm{~m}$, $3 \mathrm{H}, \mathrm{H}-3$ ', $\mathrm{PhCH}_{2}$ ), 4.15 (dd, $1 \mathrm{H}, J_{5,4}=10.3 \mathrm{~Hz}, J_{5,6}=1.4 \mathrm{~Hz}$, H-5), 4.03-3.96 (m, 3H, H-5', H-6', H-7), 3.91 (dd, 1H, J6,5 = $\left.4.7, J_{6,6},=10.9 \mathrm{~Hz}, \mathrm{H}-6^{\prime}\right), 3.88\left(\mathrm{t}, 1 \mathrm{H}, J_{3,2}=J_{3,4}=9.2 \mathrm{~Hz}\right.$, $\mathrm{H}-3), 3.67\left(\mathrm{~d}, 1 \mathrm{H}, J_{1,1}=11.0 \mathrm{~Hz}, \mathrm{H}-1^{\prime}\right), 3.51\left(\mathrm{~d}, 1 \mathrm{H}, J_{1,1}=11.0\right.$ Hz, H-1'), 3.49 (dd, 1H, $\left.J_{4,3}=9.2 \mathrm{~Hz}, J_{4,5}=10.3 \mathrm{~Hz}, \mathrm{H}-4\right)$, $3.39\left(\mathrm{dd}, 1 \mathrm{H}, J_{2,1}=3.6 \mathrm{~Hz}, J_{2,3}=9.7 \mathrm{~Hz}, \mathrm{H}-2\right), 1.96(\mathrm{~s}, 3 \mathrm{H}$, $\left.\mathrm{CH}_{3}\right), 1.84\left(\mathrm{~s}, 3 \mathrm{H}, \mathrm{CH}_{3}\right), 1.05(\mathrm{~s}, 9 \mathrm{H}, t-\mathrm{Bu}) ;{ }^{13} \mathrm{C} \mathrm{NMR} \delta 170.5$ $(\mathrm{C}=\mathrm{O}), 169.8(\mathrm{C}=\mathrm{O}), 138.6,138.4,138.2,138.1,138.0,137.9$, $135.7,135.5,133.3,133.0,129.7,129.6,128.3-127.5$ (Ar), 104.2 (C-2'), 89.0 (C-1), 83.7 (C-3'), 82.0 (C-3), 81.9 (C-4'), $81.0(\mathrm{C}-5), 79.8(\mathrm{C}-2), 77.6(\mathrm{C}-4), 75.6\left(\mathrm{PhCH}_{2}\right), 74.6$ $\left(\mathrm{PhCH}_{2}\right), 73.3\left(\mathrm{PhCH}_{2}\right), 73.1\left(\mathrm{PhCH}_{2}\right), 72.8\left(\mathrm{PhCH}_{2}\right), 72.1$ $\left(\mathrm{PhCH}_{2}\right), 71.4\left(\mathrm{C}-1^{\prime}\right), 71.1$ (C-6), 70.9 (C-5), 64.6 (C-6'), 63.2 (C-7), 26.9, 20.9, 20.8, 19.2; anal. calcd for $\mathrm{C}_{75} \mathrm{H}_{82} \mathrm{O}_{14} \mathrm{Si} \times$ $1 / 2 \mathrm{H}_{2} \mathrm{O}$ (1253.57): C, 72.38; H, 6.72; found: $\mathrm{C}, 72.51 ; \mathrm{H}, 6.36$. HRMS (ESI) calc. for $\mathrm{C}_{75} \mathrm{H}_{86} \mathrm{NO}_{14} \mathrm{Si}\left[\mathrm{M}+\mathrm{NH}_{4}\right]^{+}: 1252.5818$; found: 1252.5825 .

Data for 16-Ac: $[\alpha]_{\mathrm{D}}{ }^{20} 13.1 ;{ }^{1} \mathrm{H} \mathrm{NMR}\left(\mathrm{CDCl}_{3}\right) \delta 7.65-7.63(\mathrm{~m}$, $4 \mathrm{H}, \mathrm{Ar}), 7.34-7.20(\mathrm{~m}, 36 \mathrm{H}, \mathrm{Ar}), 6.09\left(\mathrm{~d}, 1 \mathrm{H}, J_{1,2}=3.5 \mathrm{~Hz}\right.$, $\mathrm{H}-1), 5.48$ (ddd, $1 \mathrm{H}, J=1.5 \mathrm{~Hz}, 2.9 \mathrm{~Hz}, 9.2 \mathrm{~Hz}, \mathrm{H}-6)$, 4.88-4.42 (m, 12H, $\left.\mathrm{PhCH}_{2}\right), 4.42-4.38$ (m, 2H, H-3', H-4'), $4.28\left(\mathrm{dd}, 1 \mathrm{H}, J_{7,6}=9.1 \mathrm{~Hz}, J_{7,7}=12.0 \mathrm{~Hz}, \mathrm{H}-7\right), 4.11(\mathrm{dd}, 1 \mathrm{H}$, $\left.J_{5,4}=10.1 \mathrm{~Hz}, J_{5,6}=1.3 \mathrm{~Hz}, \mathrm{H}-5\right), 4.06\left(\mathrm{dd}, 1 \mathrm{H}, J_{7,6}=3.1 \mathrm{~Hz}\right.$, $\left.J_{7,7}=12.0 \mathrm{~Hz}, \mathrm{H}-7\right), 4.02\left(\mathrm{dd}, 1 \mathrm{H}, J_{6,5}=4.2 \mathrm{~Hz}, J_{6,6}\right.$, $=11.4$ Hz, H-6'), 3.97-3.93 (m, 2H, H-3, H-5'), 3.85 (dd, 1H, $J_{6,5}=4.1$ $\mathrm{Hz}, J_{6,6}$ ' $\left.=11.4 \mathrm{~Hz}, \mathrm{H}-6^{\prime}\right), 3.74$ (d, $\left.1 \mathrm{H}, J_{1,1}=10.8 \mathrm{~Hz}, \mathrm{H}-1^{\prime}\right)$, $3.53\left(\mathrm{~d}, 1 \mathrm{H}, J_{1,1}=10.8 \mathrm{~Hz}, \mathrm{H}-1^{\prime}\right), 3.48\left(\mathrm{dd}, 1 \mathrm{H}, J_{2,1}=3.5 \mathrm{~Hz}\right.$, $\left.J_{2,3}=9.7 \mathrm{~Hz}, \mathrm{H}-2\right), 3.33\left(\mathrm{dd}, 1 \mathrm{H}, J_{4,3}=8.9 \mathrm{~Hz}, J_{4,5}=10.1 \mathrm{~Hz}\right.$, $\mathrm{H}-4), 2.08\left(\mathrm{~s}, 3 \mathrm{H}, \mathrm{CH}_{3}\right), 1.95\left(\mathrm{~s}, 3 \mathrm{H}, \mathrm{CH}_{3}\right), 1.06$ (s, 9H, $t$-Bu); ${ }^{13} \mathrm{C}$ NMR $\left(\mathrm{CDCl}_{3}\right) \delta 170.3(\mathrm{C}=\mathrm{O}), 170.2(\mathrm{C}=\mathrm{O}), 138.5,138.3$, 138.3, 138.2, 137.8, 137.7, 135.6, 135.5, 133.2, 132.7, 129.7,
129.6, 128.5-127.5 (Ar), 104.6 (C-2'), 89.1 (C-1), 83.7 (C-3'), 81.6 (C-3), 81.3 (C-4'), 80.8 (C-5'), 79.8 (C-2), 76.9 (C-4), 75.6 $\left(\mathrm{PhCH}_{2}\right), 75.0\left(\mathrm{PhCH}_{2}\right), 73.5\left(\mathrm{PhCH}_{2}\right), 73.3\left(\mathrm{PhCH}_{2}\right), 72.8$ $\left(\mathrm{PhCH}_{2}\right), 71.8\left(\mathrm{PhCH}_{2}\right), 71.4\left(\mathrm{C}-1^{\prime}\right), 70.2(\mathrm{C}-5), 69.0(\mathrm{C}-6)$, 64.8 (C-7), 63.7 (C-6'), 26.9, 21.0, 20.8, 19.2; anal. calcd for $\mathrm{C}_{75} \mathrm{H}_{82} \mathrm{O}_{14} \mathrm{Si} \times 1 / 2 \mathrm{H}_{2} \mathrm{O}$ (1253.57): C, 72.38; H, 6.72; found: $\mathrm{C}$, 72.34; H, 6.29. HRMS (ESI) calc. for $\mathrm{C}_{75} \mathrm{H}_{86} \mathrm{NO}_{14} \mathrm{Si}[\mathrm{M}+$ $\left.\mathrm{NH}_{4}\right]^{+}:$1252.5818; found: 1252.5819 .

\section{Supporting Information}

\section{Supporting Information File 1}

The ${ }^{1} \mathrm{H}$ and ${ }^{13} \mathrm{C}$ NMR spectra of all new compounds

(9-16Ac).

[http://www.beilstein-journals.org/bjoc/content/

supplementary/1860-5397-10-124-S1.pdf]

\section{Acknowledgements}

The support from Grant: POIG.01.01.02-14-102/09 (partfinanced by the European Union within the European Regional Development Fund) and N N204 187439 (from the Ministry of Science and Higher Education) is acknowledged.

\section{References}

1. Ooi, T.; Maruoka, K. Angew. Chem., Int. Ed. 2007, 46, 4222-4266. doi:10.1002/anie.200601737

2. Rapi, Z.; Démuth, B.; Keglevich, G.; Grün, A.; Drahos, L.; Sóti, P. L.; Bakó, P. Tetrahedron: Asymmetry 2014, 25, 141-147. doi:10.1016/j.tetasy.2013.12.007

3. Zhang, X. X.; Bradshaw, J. S.; Izatt, R. M. Chem. Rev. 1997, 97, 3313-3362. doi:10.1021/cr960144p

4. Cheng, C.; Cai, Z.; Peng, X.-S.; Wong, H. N. C. J. Org. Chem. 2013, 78, 8562-8573. doi:10.1021/jo401240k

5. Jarosz, S.; Listkowski, A. Curr. Org. Chem. 2006, 10, 643-662. doi:10.2174/138527206776359702

6. Bako, P.; Keglevich, G.; Rapi, Z.; Toke, L. Curr. Org. Chem. 2012, 16, 297-304. doi:10.2174/138527212799499877

7. Jarosz, S.; Potopnyk, M. A.; Kowalski, M. Sucrose as chiral platform in the synthesis of macrocyclic receptors. In Carbohydrate Chemistry-Chemical and Biological Approaches; Rauter, A. P.; Lindhorst, T.; Queneau, Y., Eds.; RSC Publishing, 2014; Vol. 40, pp 236-256. doi:10.1039/9781849739986-00236 See for a recent review.

8. Jarosz, S.; Listkowski, A. Can. J. Chem. 2006, 84, 492-496. doi:10.1139/v06-035

9. Jarosz, S.; Listkowski, A.; Lewandowski, B. Phosphorus, Sulfur Silicon Relat. Elem. 2009, 184, 1285-1295. doi:10.1080/10426500902856370

10. Queneau, Y.; Jarosz, S.; Lewandowski, B.; Fitremann, J. Adv. Carbohydr. Chem. Biochem. 2007, 61, 217-292. doi:10.1016/S0065-2318(07)61005-1

11. Jarosz, S.; Lewandowski, B. Carbohydr. Res. 2008, 343, 965-969. doi:10.1016/j.carres.2008.01.016 
12. Potopnyk, M. A.; Lewandowski, B.; Jarosz, S. Tetrahedron: Asymmetry 2012, 23, 1474-1479. doi:10.1016/j.tetasy.2012.10.003

13. Lewandowski, B.; Jarosz, S. Chem. Commun. 2008, 6399-6401. doi:10.1039/b816476b

14. Potopnyk, M. A.; Jarosz, S. Eur. J. Org. Chem. 2013, 5117-5126. doi:10.1002/ejoc.201300427

15. Lewandowski, B.; Jarosz, S. Org. Lett. 2010, 12, 2532-2535. doi:10.1021/ol100749m

16. Jarosz, S. J. Carbohydr. Chem. 2001, 20, 93-107. doi:10.1081/CAR-100103951

17. Jarosz, S.; Mach, M. J. Chem. Soc., Perkin Trans. 1 1998, 3943-3948. doi:10.1039/a807190j

18. Jarosz, S. Curr. Org. Chem. 2008, 12, 985-994. doi:10.2174/138527208785161187

19. Jarosz, S.; Mach, M.; Frelek, J. J. Carbohydr. Chem. 2000, 19, 693-715. doi:10.1080/07328300008544111

20. Mach, M.; Jarosz, S. J. Carbohydr. Chem. 2001, 20, 411-424. doi:10.1081/CAR-100105713

21 . Synthesis of this aldehyde was already reported by us as intermediate in the preparation of the uronate 8 (see ref. [8]).

22. Makosza, M.; Fedorynski, M. Adv. Catal. 1987, 35, 375-422. doi:10.1016/S0360-0564(08)60097-8

23. Makosza, M. Pure Appl. Chem. 2000, 72, 1399-1403. doi:10.1351/pac200072071399

24. Makosza, M.; Fedoryński, M. Catal. Rev.: Sci. Eng. 2003, 45, 321-367. doi:10.1081/CR-120025537

25. Jarosz, S. Carbohydr. Res. 1988, 183, 201-207. doi:10.1016/0008-6215(88)84074-6

26. Cha, J. K.; Christ, W. J.; Kishi, Y. Tetrahedron 1984, 40, 2247-2255. doi:10.1016/0040-4020(84)80008-3

27. Frelek, J.; Pakulski, Z.; Zamojski, A. Tetrahedron: Asymmetry 1996, 7, 1363-1372. doi:10.1016/0957-4166(96)00153-X

28. Frelek, J.; Ikekawa, N.; Takatsuto, S.; Snatzke, G. Chirality 1997, 9 , 578-582.

doi:10.1002/(SICI)1520-636X(1997)9:5/6<578::AID-CHIR27>3.0.CO;2$\mathrm{K}$

29. Frelek, J.; Klimek, A.; Ruśkowska, P. Curr. Org. Chem. 2003, 7, 1081-1104. doi:10.2174/1385272033486576

30. Jawiczuk, M.; Górecki, M.; Suszczyńska, A.; Karchier, M.; Jaźwiński, J.; Frelek, J. Inorg. Chem. 2013, 52, 8250-8263. doi:10.1021/ic401170m

31. Biela, A.; Oulaïdi, F.; Gallienne, E.; Górecki, M.; Frelek, J.; Martin, O. R. Tetrahedron 2013, 69, 3348-3354. doi:10.1016/j.tet.2012.12.082

32. Schönemann, W.; Gallienne, E.; Ikeda-Obatake, K.; Asano, N.; Nakagawa, S.; Kato, A.; Adachi, I.; Górecki, M.; Frelek, J.; Martin, O. R. ChemMedChem 2013, 8, 1805-1817. doi:10.1002/cmdc.201300327

\section{License and Terms}

This is an Open Access article under the terms of the Creative Commons Attribution License

(http://creativecommons.org/licenses/by/2.0), which permits unrestricted use, distribution, and reproduction in any medium, provided the original work is properly cited.

The license is subject to the Beilstein Journal of Organic Chemistry terms and conditions:

(http://www.beilstein-journals.org/bjoc)

The definitive version of this article is the electronic one which can be found at:

doi:10.3762/bjoc. 10.124 